\title{
Superexchange pathways stabilize the magnetic coupling of MnPc with Co in a spin interface mediated by graphene
}

\author{
Giulia Avvisati, ${ }^{1, *}$ Pierluigi Gargiani, ${ }^{2}$ Pierluigi Mondelli, ${ }^{1, \dagger}$ Francesco Presel,,${ }^{3, \dagger}$ \\ Alessandro Baraldi, ${ }^{3,4,5}$ and Maria Grazia Betti ${ }^{1}$ \\ ${ }^{1}$ Physics Department, Sapienza University of Rome, Piazzale Aldo Moro 5, I-00185 Rome, Italy \\ ${ }^{2}$ ALBA synchrotron Light Source, Carrer de la Llum 2-26, E-08290 Barcelona, Spain \\ ${ }^{3}$ Physics Department, University of Trieste, Via Valerio 2, I-34127 Trieste, Italy \\ ${ }^{4}$ Elettra Sincrotrone Trieste, S.S. 14 Km 163.5, I-34149 Basovizza, Trieste, Italy \\ ${ }^{5}$ IOM-CNR, S.S. $14 \mathrm{Km}$ 163.5, I-34149 Basovizza, Trieste, Italy
}

(Received 22 June 2018; published 6 September 2018)

\begin{abstract}
We investigate the magnetic response of a spin interface constituted by MnPc molecules adsorbed on graphene/Co and its robustness against thermal fluctuations by $\mathrm{x}$-ray magnetic circular dichroism. Elementselective hysteresis loops reveal a remarkable antiferromagnetic coupling between MnPc and Co that is strong both in perpendicular and in-plane magnetic orientations, thanks to the magnetic anisotropy properties and electronic configuration of MnPc. The magnetic interaction between MnPc and Co is mediated by the molecular states and the graphene $\pi$ orbitals in a superexchange mechanism that allows a strong exchange coupling while the molecular orbitals symmetries are preserved by the graphene decoupling layer. Our results show that the strength and stability of the magnetic coupling between MnPc molecules and Co layer(s), intercalated at the graphene/Ir(111) interface, is further optimized by the open $3 d$ shell of the central Mn ion. The magnetic properties are compared with analogous molecular spin interfaces with high thermal stability, paradigmatic examples to exploit in surface-supported molecular spin electronics.
\end{abstract}

DOI: 10.1103/PhysRevB.98.115412

\section{INTRODUCTION}

Molecular spin interfaces with tailored electronic and magnetic functionalities are emerging as key enablers for exploring magnetic interactions in spintronic applications [1,2]. New insights in understanding the fundamental phenomena of molecular interaction with magnetic surfaces are developing towards a new pathway to design molecular hybrid interfaces [3], but it is rare to attain both a preserved molecular magnetic state and a stable magnetic response even at room temperature $[4,5]$. Transition metal phthalocyanines ( $T M C_{32} H_{16} N_{8}$, TMPcs), square-shaped planar molecules in which a central transition metal ion, usually in the $2^{+}$oxidation state, is embedded in an organic cage, have proven to be promising molecular units in several technological applications [6]. Their easily-controllable and tunable magnetic properties depend on the configuration of the central transition metal atom [7], while the organic macrocycles ensure their highly stable and ordered assembling upon adsorption on metal surfaces [8-10]. The magnetic properties of TMPcs supported on metal surfaces may be changed by chemical modification of the molecules [11] or by strong electronic coupling with the substrate due to orbital intermixing with metallic states $[4,12]$. While the adsorption of such planar

\footnotetext{
*giulia.avvisati@uniroma1.it

$\dagger$ Present address: Merck Chemicals Ltd. - Chilworth Technical Centre, University Parkway UK-SO16 7QD Southampton.

${ }^{\ddagger}$ Present address: Technical University of Denmark, Fysikvej Building 312, DK-2800 Kgs. Lyngby.
}

molecules on a solid surface often results in a quench of the molecular spin moment due to the interaction, graphene $(\mathrm{Gr})$ can act as a buffer layer to decouple the molecular orbitals and the underlying metallic states [13,14], preserving the magnetic states of the TMPc molecules [15]. The Gr layer can also act as a mediator of the magnetic interaction, either by a direct coupling [16] or by opening an effective superexchange channel $[5,17]$.

We have recently demonstrated that the electronic configuration of the TMPc metal centers can lead to an antiferromagnetic (AFM) or weak ferromagnetic (FM) coupling with Co layer(s) intercalated under $\mathrm{Gr} / \operatorname{Ir}(111)$, depending on the dominant interaction channel [17]. The robustness of the magnetic coupling against thermal fluctuations is strongly related to different superexchange pathways and to the relative orientation between the molecule and the substrate easy magnetization axes $[17,18]$. Among all TMPcs, MnPc exhibits the highest Curie temperature (in its $\beta$ polymorph) [19] and easy-axis magnetic anisotropy energy [20], making it the ideal candidate to further optimize the magnetic coupling and the robustness against thermal fluctuations of such molecular spin interfaces.

We herewith report on the strength and stability of the magnetic coupling between $\mathrm{MnPc}$ molecules and Cointercalated Gr, for a single and few Co layer(s), by means of x-ray absorption spectroscopy (XAS) and magnetic circular dichroism (XMCD). In particular, we have investigated both the highly corrugated moiré superstructure of Gr/1 ML Co/Ir(111) [17,21,22], exhibiting an out-of-plane easy magnetization axis and a strong perpendicular magnetic anisotropy $[21,23]$, and the flat commensurate Gr sheet on 

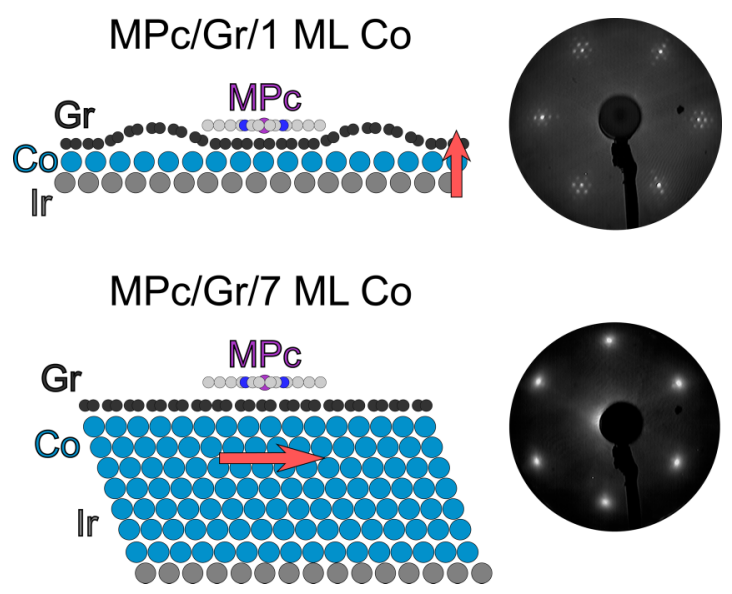

FIG. 1. Spin interface architectures: (top) moiré superstructure of Gr on $\operatorname{Ir}(111)$ upon intercalation of 1 ML Co and (bottom) flat and commensurate Gr/Ir upon 7 ML Co intercalation, as deduced by the LEED patterns reported in the right panel. At the early deposition stage, the $\mathrm{MnPc}$ molecules are trapped in the valley regions of the highly corrugated Gr/1 ML Co moiré superstructure, while MnPc adsorbs flat if the Co intercalated layers arrange commensurate with the Gr lattice.

7 ML Co [22], with the easy magnetization axis lying parallel to the surface plane [17], as displayed in Fig. 1 and in the Supplemental Material [24]. It is worth reminding that the isolated MnPc molecule has the spin-polarized metal-related molecular orbitals with projections both lying inside and protruding from the molecular plane, associated with the ${ }^{4} E_{g}$ ground state with a $b_{2 g}\left(d_{x y}\right)^{1} e_{g}\left(d_{x z, y z}\right)^{3} a_{1 g}\left(d_{z^{2}}\right)^{1} b_{1 g}\left(d_{x^{2}-y^{2}}\right)^{0}$ configuration $[7,25]$. Therefore, the MnPc molecules adsorbed on the $\mathrm{Gr} / \mathrm{Co}$ substrate have an electronic/magnetic matching for activating (both) the superexchange channels leading to an AFM (FM) alignment, recently proposed for FePc (CuPc) on Co-intercalated Gr [17]. A detailed comparison of the magnetic response of different TMPcs unveils the role of the symmetry of the molecular orbitals on the effectiveness of the activated superexchange channel(s) in these robust spin interfaces, exhibiting stability up to room temperature.

\section{EXPERIMENTAL METHODS}

XAS and XMCD measurements were carried out at the BOREAS beamline of the ALBA synchrotron radiation facility [26]. The spectra were recorded with parallel and antiparallel helicities by measuring the sample drain current, normalized with respect to the incident flux, accounted for as the drain current on a clean gold grid. The magnetic state of the sample was measured along both the easy and the hard magnetization axis, by changing the incidence angle of the x-ray beam, collinear with the applied magnetic field. In particular, the nearly-in-plane magnetic state was explored with an incidence angle of $70^{\circ}$ with respect to the surface normal, while the out-of-plane magnetic response was investigated at normal incidence. Finally, the hysteresis loops were obtained by monitoring the field dependence of the $L_{3}$ XMCD intensity, normalized at the pre-edge signal in order to cancel out any field-induced artifact. The saturation magnetization of the thick $\mathrm{MnPc}$ film was determined with a Langevin fit of the hysteresis curve, and, as also deduced by previous experiments [27], a magnetic field higher than $6 \mathrm{~T}$ is required to saturate the magnetic moment of the MnPc film. We estimate that at $6 \mathrm{~T}$ the magnetization along the easy axis is around $80 \%$ of saturation.

The $\operatorname{Ir}(111)$ surface was prepared by $2 \mathrm{keV} \mathrm{Ar}^{+}$sputtering and annealing above $1300 \mathrm{~K}$. The graphene layer was grown on the freshly-cleaned Ir surface by exposing the substrate, kept at a temperature higher than $1500 \mathrm{~K}$, to a partial ethylene $\left(C_{2} H_{4}\right)$ pressure of $1 \times 10^{-6}$ mbar. The quality of the Gr layer was verified for each experiment by monitoring the presence of sharp and bright low energy electron diffraction (LEED) spots associated to the moiré superstructure pattern. Metallic Co was then sublimated with a commercial e-beam evaporator and deposited on the $\mathrm{Gr} / \mathrm{Ir}(111)$ surface kept at room temperature. Finally, annealing the $\mathrm{Co} / \mathrm{Gr} / \mathrm{Ir}(111)$ sample at $600-800 \mathrm{~K}$ promotes Co intercalation, as also reported in Refs. $[21,22,28]$. The thickness of the intercalated Co film was predetermined with a quartz crystal microbalance and double checked with an Auger-calibrated XAS jump-edge ratio growth. The LEED patterns obtained with a definite moiré superstructure for a single Co layer and the hexagonal $1 \times 1$ pattern for the seven Co layers intercalated under graphene are reported in Fig. 1. Finally, previously purified MnPc powder was sublimated with a resistively heated quartz crucible and deposited on the $\mathrm{Gr} / \mathrm{Co} / \mathrm{Ir}(111)$ surface. The thickness of the molecular adlayer was also determined with a quartz crystal microbalance.

\section{RESULTS AND DISCUSSION}

The magnetic response of two peculiar molecular spin interfaces, constituted by MnPc molecules adsorbed (i) on the highly corrugated $\mathrm{Gr} / 1 \mathrm{ML} \mathrm{Co} / \operatorname{Ir}(111)$ and (ii) on the flat commensurate $\mathrm{Gr} / 7 \mathrm{ML} \mathrm{Co} / \mathrm{Ir}(111)$, has been investigated by $\mathrm{XMCD}$, as an element-selective probe of the magnetization. The molecular spin interface was prepared according to the procedure already described in Refs. [29,30], intercalating increasing number of Co layers under $\operatorname{Gr} / \operatorname{Ir}(111)$. The first intercalated Co layer is pseudomorphic to the $\operatorname{Ir}(111)$ surface, while, when more than four Co layers are intercalated, they arrange as HCP bulk $\mathrm{Co}(0001)$, commensurate to the $\mathrm{Gr}$ lattice [22,29], as revealed by the LEED patterns reported in Fig. 1. It is important to remind that the Co-intercalated $\mathrm{Gr}$ substrate presents a tunable easy magnetization direction: perpendicular to the surface plane for a single Co layer, in plane for larger ( $>4 \mathrm{ML}$ ) thicknesses, as deduced from the XMCD spectra at the Co $L_{2,3}$ edges, reported in the Supplemental Material [24]. The MnPc magnetic state has a high magnetic anisotropy energy with an out-of-plane magnetization direction [20], thus allowing for the evaluation of the effects of the relative orientation between the molecule and substrate easy magnetization axes in the $\mathrm{MnPc} / \mathrm{Gr} / \mathrm{Co}$ spin interfaces.

In Fig. 2 we report the Mn $L_{2,3}$ XAS and XMCD signals, measured in remanent magnetization state along the substrate easy [(a),(e)] and hard [(b),(d)] magnetization axes, for $\mathrm{MnPc}$ molecules assembled on $\mathrm{Gr} / \mathrm{Co} / \mathrm{Ir}(111)$ with outof-plane [(a),(b)] and in-plane [(d),(e)] easy magnetization 

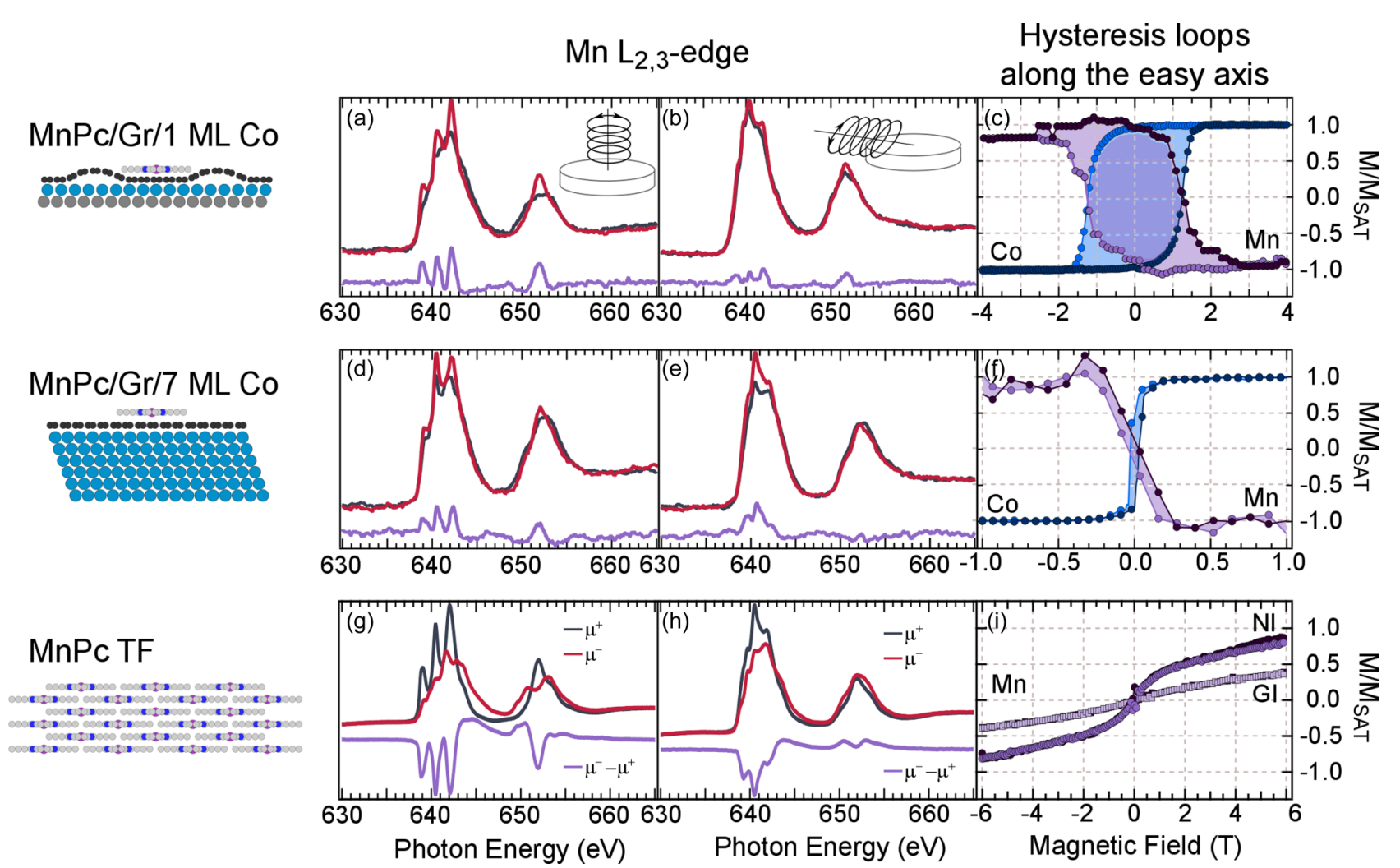

FIG. 2. XAS and XMCD of MnPc at the Mn $L_{2,3}$ edges, acquired in remanence for about $0.5 \mathrm{ML}$ of MnPc on Gr/1 ML Co [(a),(b)] and on Gr/7 ML Co [(d),(e)]. XMCD of Mn $L_{2,3}$ edges for a thick paramagnetic MnPc film, acquired with an applied magnetic field of $6 \mathrm{~T}$ [(g),(h)]. The insets display the experimental geometries: normal [(a),(d),(g)] and grazing [(b),(e),(h)] incidence. Right panel: the field dependence of the XMCD signal as acquired along the easy magnetization axis of the systems, i.e., perpendicular (c) and parallel (f) to the surface plane. All the measurements were performed at a temperature of $3 \mathrm{~K}$.

direction. A schematic view of the experimental geometry is shown in the inset. In Figs. 2(g) and 2(h) we report for comparison the XMCD signal of a paramagnetic thick MnPc film, grown flat lying on a gold (111) surface [31], measured with an applied magnetic field of $6 \mathrm{~T}$ at $3 \mathrm{~K}$. Element-selective hysteresis loops are extracted by measuring the normalized $\mathrm{XMCD}$ signal as a function of the external magnetic field applied along the easy magnetization axis of the spin interface. In order to probe the magnetic anisotropy of the MnPc molecules, we report in Fig. 2(i) the hysteresis loops collected in normal and grazing incidence on the thick MnPc film.

The XAS and XMCD spectra of the Mn ions in the thick molecular film show a rich multiplet structure (Fig. 2 bottom panel) which represents a fingerprint of the ${ }^{4} E_{g}$ ground state, corresponding to a $b_{1 g}\left(d_{x y}\right)^{1} e_{g}\left(d_{x z, y z}\right)^{3} a_{1 g}\left(d_{z^{2}}\right)^{1}$ configuration and an intermediate $S=\frac{3}{2}$ spin state [13,25,27,31-34], characterized by the coexistence of empty molecular orbitals with both out-of-plane and in-plane symmetry. The spectral lineshape, even if with subtle differences in the relative intensity of the features, is preserved for $\mathrm{MnPc}$ on $\mathrm{Gr}$ intercalated with 1 and 7 ML Co (Fig. 2 top and central panels), suggesting that the ground state configuration is not heavily altered upon adsorption. It is worth highlighting that the magnetic state of the spin interface is determined by the Co layer(s) and by the coupling of the $\mathrm{Mn}^{2+}$ ions with it, as the MnPc molecules do not have a remanent magnetization per se. Indeed, both the magnetic coercivity (see hysteresis loops, Fig. 2 right panel) and the easy magnetization axis (out-of-plane for the $\mathrm{MnPc} / \mathrm{Gr} / 1 \mathrm{ML} \mathrm{Co}$ and in-plane for the $\mathrm{MnPc} / \mathrm{Gr} / 7 \mathrm{ML}$ Co samples) of the spin interface reflect that of the Co layer(s).

The spin and orbital contribution to the MnPc magnetic moment cannot be easily deduced by applying the sum rules $[35,36]$ to the XMCD signals, due to the small spin-orbit splitting compared to the $2 p 3 d$ Coulomb exchange interaction $[37,38]$, and to the strong AFM coupling, inhibiting the Mn ions magnetic saturation. However, the similarity of the XAS and XMCD line shapes for the thick film and the MnPc sitting on Gr suggests that the orbital intermixing with the substrate only slightly influences the Mn empty orbitals and the effective $\mathrm{Mn}^{2+}$ magnetic moment. Thus, the graphene layer electronically decouples the spin interface, while a remarkable AFM coupling is present with an induced magnetic remanence of the Mn ions at $3 \mathrm{~K}$.

Recently, a detailed theoretical investigation of the interaction process for $\mathrm{FePc}$ and $\mathrm{CuPc}$ on $\mathrm{Gr} / \mathrm{Co}$ substrates unveiled identical adsorption energy landscapes and geometries for both molecules, together with similar distances between the central transition metal atoms and the underlying Co layers [17]. The assembling geometry is driven by the interaction between the organic macrocycles with the Gr layer, while the interaction of the metal-related molecular orbitals protruding out of the molecular plane with the underlying $\mathrm{Gr} / \mathrm{Co}$ interface is weak, as manifested by the subtle intensity decrease of the related XAS peaks [29]. 


\section{$180^{\circ}$ superexchange: AFM coupling}

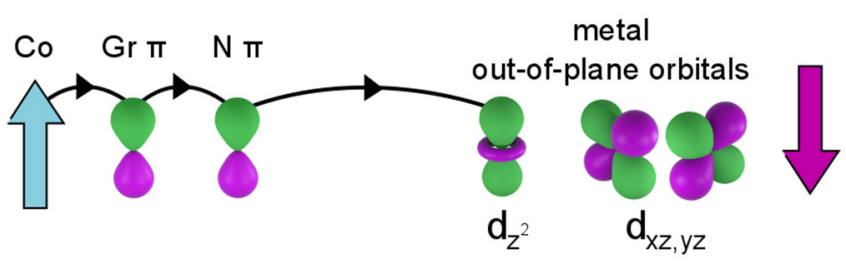

$90^{\circ}$ superexchange: $\mathrm{FM}$ coupling

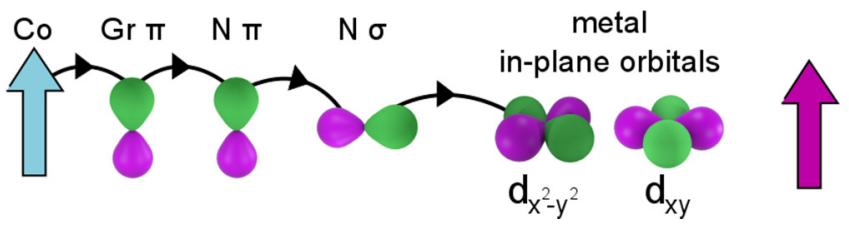

FIG. 3. The $180^{\circ}$ (top) and $90^{\circ}$ (bottom) superexchange paths are sketched, highlighting the symmetry of the molecular and graphene orbitals involved in the steps of the activated exchange channel.

Given that the central transition metal ion plays a negligible role in driving the structural arrangement of the molecular layer, we can presume that $\mathrm{MnPc}$ molecules adsorb with a similar geometry as compared with FePc and CuPc spin interfaces. Assuming identical structural configurations, only the symmetry of the molecular orbitals involved in the interaction with the extended states of Co-intercalated graphene determines the sign of the MnPc-Co magnetic coupling. The magnetization cycles of the $\mathrm{MnPc}$ molecules adsorbed on the graphene/Co substrate (Fig. 2, right panel) reveal a definite AFM alignment for all the Co intercalated configurations, consistent with what recently reported in the case of FePc molecules assembled on the same surfaces [17].

More in detail, when TMPc molecules are directly adsorbed on magnetic surfaces, they couple ferromagnetically, due to the (in)direct exchange interaction between the magnetic substrate and the central transition metal ion $[3,8,39,40]$. However, when a paramagnetic buffer layer, e.g., oxygen or graphene, is interposed between the molecules and the substrate, more complex superexchange channels can be triggered, generally favoring an AFM alignment $[5,41,42]$ but with a weaker interaction and without a detectable magnetic remanence at room temperature. Nevertheless, it was recently proposed [17] that the sign of the magnetic coupling can be modified by accurately tuning the superexchange mechanism. In particular, a $180^{\circ}$ superexchange interaction, mostly involving the out-of-plane-symmetric molecular orbitals $\left(d_{z^{2}}, d_{x z, y z}\right)$ [43], is responsible for the graphenemediated FePc-Co AFM coupling [17]. On the contrary, by selecting a magnetically polarized molecular orbital with a dominant in-plane symmetry, as for the CuPc molecule, a weaker $90^{\circ}$ superexchange path is triggered, with the magnetic coupling being transferred through the $\mathrm{N} \pi$ and the $\mathrm{N} \sigma$ orbitals, strongly hybridized with the $d_{x^{2}-y^{2}}$ metal state (see Fig. 3) [17,44]. The MnPc molecule, with a ${ }^{4} E_{g}$ ground state, has three half-filled metal-related molecular orbitals, exhibiting both in-plane $\left(b_{2 g} / d_{x y}, e_{g} / d_{x z, y z}\right)$ and out-of-plane $\left(e_{g} / d_{x z, y z}, a_{1 g} / d_{z^{2}}\right)$ projections, hence both superexchange channels can be activated. The unambiguous antiferromagnetic alignment, demonstrated by the data reported in Fig. 2 for both perpendicular and in-plane magnetic anisotropy of the $\mathrm{Gr} / \mathrm{Co}$ substrate, indicates that the superexchange path at $180^{\circ}$ dominates the coupling, similar to that observed for $\mathrm{FePc} / \mathrm{Gr} / \mathrm{Co}$ [17]. Moreover, in contrast to $\mathrm{FePc} / \mathrm{Gr} / \mathrm{Co}$, the out-of-plane easy axis of the MnPc molecule strengthens the magnetic coupling to perpendicular $\mathrm{Gr} / \mathrm{Co}$ layer. Other interaction pathways, such as a direct coupling between the $\mathrm{Gr} \pi$ orbitals, carrying a small Co-induced magnetic moment $[17,21,45]$, and the Mn-related molecular orbitals protruding out of the molecular plane, can also contribute to the Mn-Co magnetic coupling. However, due to the low magnetic polarization of the Gr sheet and the fairly large TMPcs-Gr distance [17], a direct channel would have a negligible effect. Other interaction mechanisms that could contribute to the TMPc-Co magnetic coupling are of dipolar nature. The field generated by the Co layer is calculated [46] in the Supplemental Material [24], and it contributes by less than $0.05 \mathrm{~T}$ in both structural configurations, with the molecules sitting at more than $5 \AA$ from the intercalated ferromagnetic film [17]. Therefore, the contribution of dipolar interactions also plays a negligible role in our molecular spin interfaces and superexchange interactions are the dominant coupling mechanisms, effectively stabilizing the magnetic response of the $\mathrm{MnPc}$ molecules at such distances.

It is interesting to compare the temperature-dependent $\mathrm{XMCD}$ evolution of these spin interfaces with the robustness against thermal fluctuations of analogous systems reported in the literature [5,17]. In Fig. 4 the XMCD intensity is displayed as a function of temperature, for the spin interface with $\mathrm{MnPc}$ adsorbed on the highly corrugated (left) and on the flat commensurate (right) graphene-Co substrates. For both configurations a signal can be detected even at room temperature, suggesting a robust magnetic remanence against thermal fluctuations. In the right panel of Fig. 4 we present the thermal evolution of the XMCD signal intensity of $\mathrm{MnPc} / \mathrm{Gr} / 7 \mathrm{ML}$ $\mathrm{Co}$, where the easy magnetization axis of the MnPc molecules is perpendicular to that of the $\mathrm{Gr} / \mathrm{Co}$ substrate. The presence of a finite XMCD signal at RT proves an unexpected magnetic coupling between the Co layer and Mn ions, stabilized against thermal fluctuations up to room temperature, even if the molecule and substrate easy magnetization axes are not aligned. The temperature progression of the $\mathrm{Mn}$ ion magnetization and the saturation value at RT are comparable to that recorded for FePc assembled on the same substrate [17], even if the latter configuration is more favorable because both the $\mathrm{FePc}$ molecules and the substrate have an easy-plane magnetic anisotropy. A Brillouin fit $\left[B_{3 / 2}\left(E_{e x} / k_{B} T\right)\right]$ performed over the XMCD thermal evolution can give an estimation of the exchange energy, equal to $3.4 \pm 0.4 \mathrm{meV}$ for $\mathrm{MnPc}$ [47] and $2.8 \pm 0.6 \mathrm{meV}$ for FePc [17]. It is worth pointing out that, despite the large predicted out-of-plane magnetic anisotropy of MnPc molecules, the magnetic coupling with the Gr/7 ML Co substrate is not hindered by the noncollinearity between the MnPc-Co easy axis/plane, but still exhibits a dichroic signal of around $10 \%$ at RT (with respect to the magnetic response probed at $3 \mathrm{~K}$ ). On the other hand, when the TMPcs molecules are adsorbed on the Gr/1 ML Co rippled interface and the easy magnetization axes of the molecules and the substrate are aligned, the exchange coupling is aided by the 


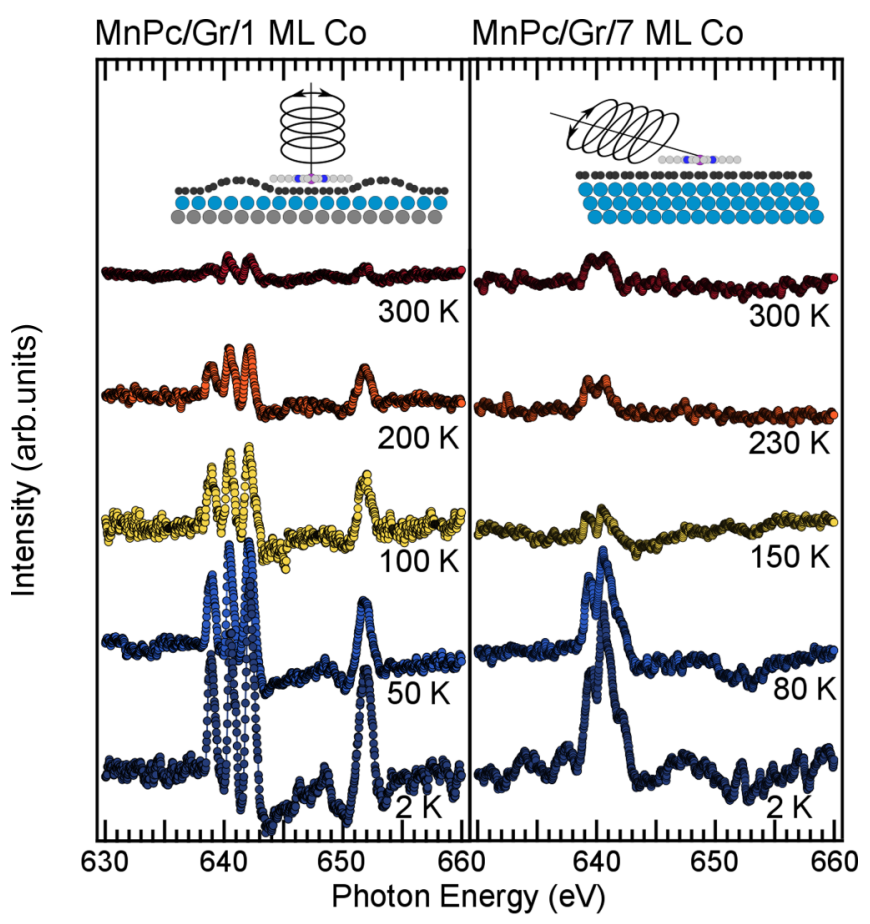

building blocks are directly deposited on a bare Co surface [48]. In the latter case, however, the exchange coupling is driven by the orbital intermixing of the molecules with the metallic magnetic states, with the formation of a hybrid metal-organic interface. In our case, the graphene buffer layer preserves the magnetic state of these planar molecules, minimizing the orbital intermixing with the underlying metallic extended states. The large adsorption distance between the metallic ion centers and the Co layers weakens the exchange interaction but preserves the magnetic identity of the components of the spin interface. In fact, despite the large TMCo distance in a TMPc/Gr/Co spin architecture, predicted to be higher than $5 \AA$ [17], the superexchange interaction mechanism stabilizes the magnetic response of the adsorbed molecular units, further optimized by accurately selecting the central TM ion.

\section{CONCLUSIONS}

The moments of paramagnetic MnPcs molecules can be aligned by a remarkable antiferromagnetic coupling to Co layer(s) intercalated under graphene. The superexchange pathway, driven by the symmetry of the orbitals carrying the magnetic moments, is mediated via the organic ligands and the electronic $\pi$ states of graphene, as recently observed for $\mathrm{FePc}$ and $\mathrm{CuPc}$ spin interfaces. The graphene spacer preserves the spin state of the TMPcs molecules adsorbed on Gr, while a slight orbital intermixing with the metal leaves their symmetry almost unaltered. The efficiency of the $\mathrm{Co}-\mathrm{Gr}-\mathrm{N}-\mathrm{TM} 180^{\circ}$ superexchange channel in driving a robust antiferromagnetic alignment between $\mathrm{MnPc}$ molecular architectures and Co layer(s), is stable up to room temperature. In addition, despite the large predicted out-of-plane magnetic anisotropy energy, the $\mathrm{Mn}^{2+}$ central metal ion exhibits a magnetic activity at RT both when the molecule-substrate easy magnetization axes are aligned $(\mathrm{MnPc} / \mathrm{Gr} / 1 \mathrm{ML} \mathrm{Co})$ and perpendicular ( $\mathrm{MnPc} / \mathrm{Gr} / 7 \mathrm{ML} \mathrm{Co}$ ) to each other. MnPc molecules are thus ideal candidates to optimize the magnetic response with both perpendicular and in-plane magnetic orientations, thanks to their relatively high magnetic moment and out-of-plane easy axis.

A new platform is now open to design magnetic molecules and spin interface architectures with optimized magnetic response at room temperature, preserving the magnetic identity of each component thanks to the insertion of the graphene layer. These tunable magnetic building blocks can play an unprecedented role in the future of molecular spintronics.

magnetic anisotropy term determining an exchange energy of $6.2 \pm 0.5 \mathrm{meV}$, the highest value obtained for this class of systems [5,17]. It is worth pointing out that for $\mathrm{FePc}$ and $\mathrm{CuPc}$ adsorbed on the perpendicularly magnetized $\mathrm{Gr} / \mathrm{Co}$ interface, the XMCD signal vanishes at $200 \mathrm{~K}$ and $50 \mathrm{~K}$, respectively. In the first case due to the frustrated magnetic interaction, being the easy molecule and substrate magnetization directions perpendicular to each other, in the latter due to the lesseffective $90^{\circ}$ FM superexchange path. Certainly, these values for the Mn-Co exchange energy are more than one order of magnitude smaller than those obtained when the molecular

\section{ACKNOWLEDGMENTS}

These XMCD experiments were performed at the BOREAS beamline at ALBA Synchrotron (Barcelona) with the collaboration of ALBA staff and CALIPSOplus (Grant No. 730872) funding. G.A. and M.G.B. acknowledge fundings from Sapienza University of Rome. P.G. acknowledges funding from the Spanish MINECO (Grants No. FIS201678591-C3-1-R and No. MAT2014-59315-R). 
[1] S. Sanvito, Nat. Phys. 6, 562 (2010).

[2] M. Cinchetti, V. A. Dediu, and L. E. Hueso, Nat. Mater. 16, 507 (2017).

[3] H. Wende, M. Bernien, J. Luo, C. Sorg, N. Ponpandian, J. Kurde, J. Miguel, M. Piantek, X. Xu, P. Eckhold et al., Nat. Mater. 6, 516 (2007).

[4] E. Annese, F. Casolari, J. Fujii, and G. Rossi, Phys. Rev. B 87, 054420 (2013).

[5] C. F. Hermanns, K. Tarafder, M. Bernien, A. Krüger, Y.-M. Chang, P. M. Oppeneer, and W. Kuch, Adv. Mater. 25, 3473 (2013).

[6] D. Dini and M. Hanack, J. Porphyr. Phthalocya. 08, 915 (2004).

[7] J. Bartolomé, C. Monton, and I. K. Schuller, Molecular Magnets: Physics and Applications, edited by J. Bartolomé, F. Luis, and J. F. Fernández (Springer, Berlin, Heidelberg, 2014), pp. 221-245.

[8] A. Lodi Rizzini, C. Krull, A. Mugarza, T. Balashov, C. Nistor, R. Piquerel, S. Klyatskaya, M. Ruben, P. Sheverdyaeva, P. Moras et al., Surf. Sci. 630, 361 (2014).

[9] M. G. Betti, P. Gargiani, C. Mariani, R. Biagi, J. Fujii, G. Rossi, A. Resta, S. Fabris, S. Fortuna, X. Torrelles et al., Langmuir 28, 13232 (2012).

[10] S. Fortuna, P. Gargiani, M. G. Betti, C. Mariani, A. Calzolari, S. Modesti, and S. Fabris, J. Phys. Chem. C 116, 6251 (2012).

[11] P. Gambardella, S. Stepanow, A. Dmitriev, J. Honolka, F. M. F. de Groot, M. Lingenfelder, S. S. Gupta, D. D. Sarma, P. Bencok, S. Stanescu et al., Nat. Mater. 8, 189 (2009).

[12] P. Gargiani, G. Rossi, R. Biagi, V. Corradini, M. Pedio, S. Fortuna, A. Calzolari, S. Fabris, J. C. Cezar et al., Phys. Rev. B 87, 165407 (2013).

[13] J. Uihlein, H. Peisert, H. Adler, M. Glaser, M. Polek, R. Ovsyannikov, M. Bauer, and T. Chassé, J. Phys. Chem. C 118, 28671 (2014).

[14] M. Scardamaglia, S. Lisi, S. Lizzit, A. Baraldi, R. Larciprete, C. Mariani, and M. G. Betti, J. Phys. Chem. C 117, 3019 (2013).

[15] S. Lisi, P. Gargiani, M. Scardamaglia, N. B. Brookes, V. Sessi, C. Mariani, and M. G. Betti, J. Phys. Chem. Lett. 6, 1690 (2015).

[16] A. Candini, V. Bellini, D. Klar, V. Corradini, R. Biagi, V. De Renzi, K. Kummer, N. B. Brookes, U. del Pennino, H. Wende et al., J. Phys. Chem. C 118, 17670 (2014).

[17] G. Avvisati, C. Cardoso, D. Varsano, A. Ferretti, P. Gargiani, and M. G. Betti, Nano Lett. 18, 2268 (2018).

[18] V. Corradini, A. Candini, D. Klar, R. Biagi, V. De Renzi, A. Lodi Rizzini, N. Cavani, U. del Pennino, S. Klyatskaya, M. Ruben, E. Velez-Fort, K. Kummer, N. B. Brookes, P. Gargiani, H. Wende, and M. Affronte, Nanoscale 10, 277 (2018).

[19] H. Miyoshi, H. Ohya-Nishiguchi, and Y. Deguchi, Bull. Chem. Soc. Jpn. 46, 2724 (1973).

[20] J. Wang, Y. Shi, J. Cao, and R. Wu, Appl. Phys. Lett. 94, 122502 (2009).

[21] R. Decker, J. Brede, N. Atodiresei, V. Caciuc, S. Blügel, and R. Wiesendanger, Phys. Rev. B 87, 041403 (2013).

[22] D. Pacilé, S. Lisi, I. Di Bernardo, M. Papagno, L. Ferrari, M. Pisarra, M. Caputo, S. K. Mahatha, P. M. Sheverdyaeva, P. Moras et al., Phys. Rev. B 90, 195446 (2014).
[23] H. Yang, A. D. Vu, A. Hallal, N. Rougemaille, J. Coraux, G. Chen, A. K. Schmid, and M. Chshiev, Nano Lett. 16, 145 (2016).

[24] See Supplemental Material at http://link.aps.org/supplemental/ 10.1103/PhysRevB.98.115412 for further details on the Co-intercalated Gr substrate and the generated dipolar field.

[25] I. E. Brumboiu, R. Totani, M. de Simone, M. Coreno, C. Grazioli, L. Lozzi, H. C. Herper, B. Sanyal, O. Eriksson, C. Puglia et al., J. Phys. Chem. A 118, 927 (2014).

[26] A. Barla, J. Nicolás, D. Cocco, S. M. Valvidares, J. HerreroMartín, P. Gargiani, J. Moldes, C. Ruget, E. Pellegrin, and S. Ferrer, J. Synchrotron Radiat. 23, 1507 (2016).

[27] T. Kataoka, Y. Sakamoto, Y. Yamazi, V. R. Sing, A. Fujimori, Y. Takeda, T. Ohkochi, S.-I. Fujimori, T. Okane, Y. Sitoh et al., Solid State Commun. 152, 806 (2012).

[28] F. Presel, N. Jabeen, M. Pozzo, D. Curcio, L. Omiciuolo, P. Lacovig, S. Lizzit, D. Alfè, and A. Baraldi, Carbon 93, 187 (2015).

[29] G. Avvisati, S. Lisi, P. Gargiani, A. Della Pia, O. De Luca, D. Pacilé, C. Cardoso, D. Varsano, D. Prezzi, A. Ferretti et al., J. Phys. Chem. C 121, 1639 (2017).

[30] G. Avvisati, P. Mondelli, P. Gargiani, and M. G. Betti, Appl. Surf. Sci. 432, 2 (2018).

[31] P. Gargiani, S. Lisi, G. Avvisati, P. Mondelli, S. Fatale, and M. G. Betti, J. Chem. Phys. 147, 134702 (2017).

[32] M.-S. Liao, J. D. Watts, and M.-J. Huang, Inorg. Chem. 44, 1941 (2005).

[33] F. Petraki, H. Peisert, F. Latteyer, U. Aygül, A. Vollmer, and T. Chassé, J. Phys. Chem. C 115, 21334 (2011).

[34] T. Kroll, R. Kraus, R. Schönfelder, V. Y. Aristov, O. V. Molodtsova, P. Hoffmann, and M. Knupfer, J. Chem. Phys. 137, 054306 (2012).

[35] B. T. Thole, P. Carra, F. Sette, and G. van der Laan, Phys. Rev. Lett. 68, 1943 (1992).

[36] P. Carra, B. T. Thole, M. Altarelli, and X. Wang, Phys. Rev. Lett. 70, 694 (1993).

[37] C. Piamonteze, P. Miedema, and F. M. F. de Groot, Phys. Rev. B 80, 184410 (2009).

[38] Y. Teramura, A. Tanaka, and T. Jo, J. Phys. Soc. Jpn. 65, 1053 (1996).

[39] F. Djeghloul, F. Ibrahim, M. Cantoni, M. Bowen, L. Joly, S. Boukari, P. Ohresser, F. Bertran, P. Le Fèvre, P. Thakur et al., Sci. Rep. 3, 1272 (2015).

[40] C. Wäckerlin, K. Tarafder, D. Siewert, J. Girovsky, T. Hählen, C. Iacovita, A. Kleibert, F. Nolting, T. A. Jung, P. M. Oppeneer et al., Chem. Sci. 3, 3154 (2012).

[41] D. Klar, B. Brena, H. C. Herper, S. Bhandary, C. Weis, B. Krumme, C. Schmitz-Antoniak, B. Sanyal, O. Eriksson, and H. Wende, Phys. Rev. B 88, 224424 (2013).

[42] M. Bernien, J. Miguel, C. Weis, M. E. Ali, J. Kurde, B. Krumme, P. M. Panchmatia, B. Sanyal, M. Piantek, P. Srivastava et al., Phys. Rev. Lett. 102, 047202 (2009).

[43] J. Bartolomé, F. Bartolomé, L. M. García, G. Filoti, T. Gredig, C. N. Colesniuc, I. K. Schuller, and J. C. Cezar, Phys. Rev. B 81, 195405 (2010).

[44] A. Rosa and E. J. Baerends, Inorg. Chem. 33, 584 (1994).

[45] H. Vita, S. Böttcher, P. Leicht, K. Horn, A. B. Shick, and F. Máca, Phys. Rev. B 90, 165432 (2014). 
[46] A. Lodi Rizzini, C. Krull, T. Balashov, J. J. Kavich, A. Mugarza, P. S. Miedema, P. K. Thakur, V. Sessi, S. Klyatskaya, M. Ruben et al., Phys. Rev. Lett. 107, 177205 (2011).

[47] This value has been obtained by imposing a $J=3 / 2$ spin state in the Brillouin fit. Indeed, in agreement with what was theoretically predicted for $\mathrm{FePc}$ and $\mathrm{CuPc}$, the spin state of the molecule is not altered upon adsorption. Therefore, we assumed that the same applies for the $\mathrm{MnPc} / \mathrm{Gr} / \mathrm{Co}$ spin interface.

[48] M. Bernien, X. Xu, J. Miguel, M. Piantek, P. Eckhold, J. Luo, J. Kurde, W. Kuch, K. Baberschke, H. Wende, and P. Srivastava, Phys. Rev. B 76, 214406 (2007). 